\title{
Wide area measurements based fault detection and location method for transmission lines
}

\author{
Wen Fan and Yuan Liao*
}

\begin{abstract}
Electric power grids are critical infrastructure for delivering energy from generation stations to load centers. To maximize utilization of assets, it is desirable to increase the power transferred over transmission systems. Reliable protection of transmission systems is essential for safeguarding the integrity and reliability of the power grid. Distance protection is the most widely used scheme for protecting transmission lines. Most existing protection systems use local measurements to make a decision while pilot protection is used in some circumstances. Distance protection may fail under stressed operating conditions, which could lead to cascading faults. This paper proposes a system integrity protection scheme by utilizing wide area measurements. The scheme partitions the system into subnetworks or protection zones and employs current measurements to derive a fault identification vector indicating the faulted zone. Then the fault location is pinpointed based on wide area measurements and network data. The proposed method is able to deal with multiple, simultaneous faults, and is applicable to both transposed and untransposed lines. Evaluation studies based on simulation studies are presented.
\end{abstract}

Keywords: System integrity protection scheme, Fault location, Transmission systems

\section{Introduction}

The electric power is delivered from generation sources to distribution system through the transmission system. Faults occurring on any section of the transmission system will disturb or even interrupt the power delivery [1-3]. Thus, when faults occur on the network, it is vital to quickly and accurately pinpoint and isolate the faulted section so that the remaining network can continue operation. Existing protection systems including distance protection usually employ local measurements to make a tripping decision. Relay coordination for desired selectivity becomes complex and challenging for meshed transmission systems due to changing loading conditions.

System integrity protection schemes (SIPS) aim to enhance protection system to increase system stability, security and integrity by making use of measurements collected from different locations in the network based on analytical techniques [4-8]. Synchronized phasor measurement techniques from phasor measurement

* Correspondence: yuan.liao@uky.edu

Department of Electrical and Computer Engineering, University of Kentucky, Lexington, KY 40506, USA 
required. Fault location algorithms that utilize two-terminal measurements are proposed in [10, 11]. Fault location techniques that utilize traveling waves generated by faults are proposed in [12-14]. Artificial neural network is used to pinpoint the fault location in $[15,16]$.

Double-circuit transmission line is known to be complicated due to mutual coupling effects, and some authors discuss fault location methods that deal with double-circuit lines. Kawady and Stenzel [17] provide a method depends on the data extracted from one end of the line. Factors such as unknown fault resistance that can impact the fault location estimation accuracy are compensated. Article [18] proposes an algorithm based on two-terminal unsynchronized voltage and current measurements.

In recent years, the rapid advances in the communication and measurement technologies such as PMU have made possible the wide area measurement system (WAMS) [4, 19, 20]. Methods to locate the fault based on sparsely located wide area synchronized measurements are presented in [21-24]. Particularly, in [22], traveling wave technique is utilized to locate the fault. In [23], the fault location is formulated as a sparse estimation problem, which can be solved by using Lasso Regression. In [24], A PMU placement scheme is described to achieve fault location observability. However, like any other systems, WAMS failure may occur as well. A quantified reliability evaluation method for the WAMS using Markov model is available in [25]. Moreover, the wide area measurements need to be accurate in order to locate the fault. The authors of [26] describe a technique to detect bad wide area measurement by using a pre-estimation filter. In [27], a linear method for identifying measurement errors has been described. Also, the number of meter placement is limited due to various reasons such as the lack of funding. Optimal PMU placement and its required communication infrastructure are co-optimized in [28].

However, there is still a need to develop fault location algorithm to handle multiple simultaneous faults. This paper proposes an algorithm to locate simultaneous faults in the transmission system using wide area measurements. In particular, cross-country faults will be examined in the case studies. The proposed algorithm utilizes distributed parameter line model and fully considers the effect of shunt capacitance of the transmission lines.

The rest of this paper is organized as follows: Section 2 describes the method of fault zone identification. Section 3 presents the proposed fault location algorithm for multiple simultaneous faults. Section 4 reports simulation results of fault zone identification method and fault location algorithm applied to a 27-bus transmission system. Section 5 provides conclusions.

\section{Fault zone identification}

This section describes the method for identifying the fault zone in a transmission system. The method develops a fault zone identification vector (FZIV) to determine the fault zones. To obtain FZIV, connection matrix $N$ and current vector $I$ are introduced as follows.

The connection matrix $N$ represents the relationship between protection zones and currents that flow through the zone boundaries. The following steps describe the procedures for establishing $\boldsymbol{N}$.

(1) For a transmission system with $k$ protection zones and $l$ currents that flow through the zone boundaries, initialize a zero matrix $\boldsymbol{N}$ with $k$ rows and $l$ columns.

(2) If the lth current flows into the $k$ th protection zone, update $N_{k l}$ to 1 .

(3) If the lth current flows out of the $k$ th protection zone, update $N_{k l}$ to -1 .

(4) Repeat procedures $(2,3)$ until all protection zones and currents are included in $\mathbf{N}$.

The current vector $I$ consists of currents that flow through the protection zone boundaries. The currents are fundamental frequency phasors, and their direction can be selected arbitrarily. Note that the vector $I$ is a column vector, with $l$ rows.

The approach to obtain FZIV is given by (1),

$$
F Z I V=|N I|
$$

where $|$.$| denotes the magnitude of a matrix. Each elem-$ ent of $\boldsymbol{F Z I V}$ indicates whether or not a fault occurs in its relevant protection zone.

In practice, FZIV can be calculated for phase A, B, and $\mathrm{C}$, separately. For example, let $\boldsymbol{I}_{a}$ be the current vector for phase A,

$$
\boldsymbol{F Z I} \boldsymbol{V}_{a}=\left|\boldsymbol{N I} \boldsymbol{I}_{a}\right|
$$

where $\boldsymbol{F Z I} \boldsymbol{V}_{a}$ is the fault zone identification vector for phase A.

If the value of the $k t h$ element of $\boldsymbol{F Z I V} \boldsymbol{V}_{a}$ during the fault is much larger than the value preceding the fault, then the $k t h$ protection zone is determined to be a fault zone with a fault involving phase A. This approach has the advantage that FZIV is associated with each phase. That is, it provides a direct approach to identifying the fault zones and faulted phases at the same time.

Note that the fault zone identification method works for single fault as well as multiple simultaneous faults. In particular, the method is able to identify fault zones and 
fault phases for simultaneous faults involving double-circuit transmission line.

It is evident that the resolution of fault zone identification depends on the number of meters placed in the transmission system. The zones can be defined according to the boundaries whee meters are located. Generally, the more meters available, the more protection zones that can be defined and the better resolution we can obtain for the possible fault areas. In practice, protection zone partition will need to consider the location and availability of meters.

\section{Fault location}

In this section, the fault location algorithm is proposed. The algorithm aims at pinpointing fault locations for multiple simultaneous faults. The algorithm is certainly able to locate a single fault as well.

The basic idea of the proposed fault location algorithm is as follows. Without loss of generality, consider simultaneous faults that occur in a transmission system. Fictitious fault buses will be added. Then the bus impedance matrix with fault buses of the transmission system can be established. Furthermore, the measured voltages at each bus during the fault are functions of the relevant driving point impedances and transfer impedances. The driving point impedances are the principal diagonal elements of the bus impedance matrix, and the transfer impedances are the off-diagonal elements [29]. Moreover, the driving point impedances and the transfer impedances are derived in terms of the fault locations. Therefore, the measured voltages during the fault can be derived in terms of the fault locations as well. Consequently, the fault locations can be solved based on the wide area voltage measurements.

The method for deriving the driving point impedances and the transfer impedances during the fault is described in detail in Section 3.1. The fault location algorithm is proposed in Section 3.2.

\subsection{Derivation of driving point impedances and transfer impedances}

In this section, the driving point impedances and the transfer impedances during the fault will be derived. A the fault point, we add non-existent nodes, or called fictitious nodes. These nodes are called fictitious fault nodes. In contrast, the nodes at other buses are called non-fault nodes. The impedances between non-fault nodes and fault nodes will be derived first in Section 3.1.1. The impedances between fault nodes will be derived in Section 3.1.2.

Figure 1 depicts the one-line diagram of a three-phase transmission line segment. Symbols $p$ and $q$ represent the buses of the line. Bus $p$ comprises nodes $p_{1}, p_{2}$, and $p_{3}$. Bus $q$ comprises nodes $q_{1}, q_{2}$, and $q_{3}$. Define the fictitious fault bus to be $r$, consisting of nodes $r_{1}, r_{2}$, and $r_{3}$. The remaining notations in Fig. 1 are explained as follows:

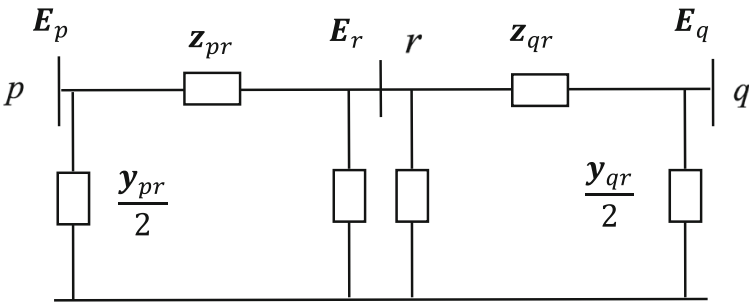

Fig. 1 A segment of a transmission system

$\boldsymbol{E}_{p}$ : node voltage vector during the fault for bus $p . \boldsymbol{E}_{p}$ $=\left[E_{p 1}, E_{p 2}, E_{p 3}\right]^{T}$, with $\mathrm{T}$ denoting vector transpose;

$\boldsymbol{E}_{q}$ : node voltage vector during the fault for bus $q . \boldsymbol{E}_{q}$ $=\left[E_{q 1}, E_{q 2}, E_{q 3}\right]^{T}$;

$\boldsymbol{E}_{r}$ : node voltage vector during the fault for bus $r$. $\boldsymbol{E}_{r}$ $=\left[E_{r 1}, E_{r 2}, E_{r 3}\right]^{T}$;

$\boldsymbol{z}_{p n}, \boldsymbol{z}_{q r}$ : the equivalent series impedance matrix of the line segment $p r$ and $q r$, respectively;

$\boldsymbol{y}_{p n} \boldsymbol{y}_{q r}$ : the equivalent shunt admittance matrix of the line segment $p r$ and $q r$, respectively.

The method is based on distributed parameter line model to accurately represent long line effects. The parameters of the equivalent PI model [30] of line segment $p r$ are as follows:

$$
\begin{aligned}
& \boldsymbol{z}_{p r}=\boldsymbol{z} \boldsymbol{B} \operatorname{diag}\left[\sinh \left(\gamma l_{p r}\right) \cdot / \boldsymbol{\gamma}\right] \boldsymbol{B}^{-1} \\
& \boldsymbol{y}_{p r}=2 \boldsymbol{B} \operatorname{diag}\left[\tanh \left(\gamma l_{p r} / 2\right) \cdot / \boldsymbol{\gamma}\right] \boldsymbol{B}^{-1} \boldsymbol{y}
\end{aligned}
$$

where,

$\operatorname{diag}($.$) : a diagonal matrix with input vector as its diag-$ onal element;

./: element-wise division;

$z$ : series impedance matrix of the line $p q$ in per unit length;

$y$ : shunt admittance matrix of the line $p q$ in per unit length;

$\boldsymbol{B}$ : eigenvector of $(\boldsymbol{y z})$;

$\gamma$ : vector consisting of $\gamma_{j}$ that is the square root of the $j$ th eigenvalue of $(y z)$.

$l_{p r}$ : length of the line segment $p r$.

Similarly, the parameters of the equivalent PI model of line segment $q r$ can be derived. It should be noted that (3) and (4) hold for both transposed and untransposed lines. Moreover, they are applicable to single-circuit lines with $j=$ $1,2,3$ as well as to double-circuit lines with $j=1,2, \ldots, 6$.

In addition, define the following variables:

$m$ : per unit fault distance from bus $p$ to the fault bus $r$; $n$ : the total number of nodes of the transmission system without counting fictitious fault nodes;

$Z_{0}$ : the bus impedance matrix in phase domain of the network preceding the fault, excluding fictitious fault nodes; 
$Z_{0, k l}$ : the element in the $k t h$ row and $l$ th column of $Z_{0}$;

$Z$ : the bus impedance matrix in phase domain of the network during the fault, including fictitious fault nodes;

$Z_{k l}$ : the element in the $k t h$ row and $l$ th column of $Z$;

Based on the definition, the size of $Z_{0}$ is $n$ by $n$. The size of $\boldsymbol{Z}$ is $\left(n+3 n_{f}\right)$ by $\left(n+3 n_{f}\right)$, where $n_{f}$ represents the total number of faults in the transmission system.

Matrix $Z_{0}$ can be readily developed following the established method in [29]. It can be shown that the first $n$ rows and $n$ columns of $Z$ are identical to $Z_{0}$, and the rest of rows and columns of $Z$ consist of driving point and transfer impedances related to the fault nodes.

\subsubsection{Derivation of the transfer impedance between a} non-fault node and fault nodes

Remove all the sources in the network shown in Fig. 1. Then inject 1 Ampere current into node $k$ [31]. Applying Kirchhoff's Current Law (KCL) at bus $r$, it is obtained that

$$
\frac{\left(\boldsymbol{y}_{p r}+\boldsymbol{y}_{q r}\right)}{2} \boldsymbol{E}_{r}+\boldsymbol{z}_{p r}^{-1}\left(\boldsymbol{E}_{r}-\boldsymbol{E}_{p}\right)+\boldsymbol{z}_{q r}^{-1}\left(\boldsymbol{E}_{r}-\boldsymbol{E}_{q}\right)=0
$$

From (5), $\boldsymbol{E}_{r}$ can be written as

$$
\boldsymbol{E}_{r}=\left[\frac{\left(\boldsymbol{y}_{p r}+\boldsymbol{y}_{q r}\right)}{2}+\boldsymbol{z}_{p r}^{-1}+\boldsymbol{z}_{q r}^{-1}\right]^{-1}\left(\boldsymbol{z}_{p r}^{-1} \boldsymbol{E}_{p}+\boldsymbol{z}_{q r}^{-1} \boldsymbol{E}_{q}\right)
$$

Based on the definition of transfer impedance, the value of the transfer impedance between a non-fault node $k$ and a fault node is equal to the voltage at the fault node, when 1 Ampere current is injected into node $k$, with all the sources in the network being removed [31]. Therefore, the values of $\boldsymbol{E}_{p}$ and $\boldsymbol{E}_{q}$ are equal to $\boldsymbol{Z}_{k p}$ and $Z_{k q}$, respectively. Hence, the transfer impedance between node $k$ and fault nodes are:

$$
\boldsymbol{Z}_{k r}=\left[\frac{\left(\boldsymbol{y}_{p r}+\boldsymbol{y}_{q r}\right)}{2}+\boldsymbol{z}_{p r}^{-1}+\boldsymbol{z}_{q r}^{-1}\right]^{-1}\left(\boldsymbol{z}_{p r}^{-1} \boldsymbol{Z}_{k p}+\boldsymbol{z}_{q r}^{-1} \boldsymbol{Z}_{k q}\right)
$$

where,

$Z_{k r}=\left[Z_{k r_{1}}, Z_{k r_{2}}, Z_{k r_{3}}\right]^{T}$, with $\mathrm{T}$ denotes vector transpose;

$Z_{k p}=\left[Z_{k p_{1}}, Z_{k p_{2}}, Z_{k p_{3}}\right]^{T}$;

$Z_{k q}=\left[Z_{k q_{1}}, Z_{k q_{2}}, Z_{k q_{3}}\right]^{T}$;
$Z_{k r_{1}}, Z_{k r_{2}}, Z_{k r_{3}}$ : the transfer impedance between node $k$ and fault node $r_{1}, r_{2}$, and $r_{3}$, respectively;

$Z_{k p_{1}}, Z_{k p_{2}}, Z_{k p_{3}}$ : the transfer impedance between node $k$ and node $p_{1}, p_{2}$, and $p_{3}$, respectively;

$Z_{k q_{1}}, Z_{k q_{2}}, Z_{k q_{3}}$ : the transfer impedance between node $k$ and node $q_{1}, q_{2}$, and $q_{3}$, respectively.

Note that $Z_{k p 1}, Z_{k q 1}$ and so on are elements of the bus impedance matrix of the prefault network. Furthermore, $\mathbf{y}_{p n}, \mathbf{y}_{q n}, \mathbf{z}_{p n}$ and $\mathbf{z}_{q r}$ are functions of the fault location $m$. Therefore, the transfer impedance $Z_{k r}$ is a function of the fault location $m$ as well.

The prefault voltage at fictitious fault nodes can be calculated using the prefault terminal voltages based on (6):

$$
\boldsymbol{E}_{r}^{0}=\left[\frac{\left(\boldsymbol{y}_{p r}+\boldsymbol{y}_{q r}\right)}{2}+\boldsymbol{z}_{p r}^{-1}+\boldsymbol{z}_{q r}^{-1}\right]^{-1}\left(\boldsymbol{z}_{p r}^{-1} \boldsymbol{E}_{p}^{0}+\boldsymbol{z}_{q r}^{-1} \boldsymbol{E}_{q}^{0}\right)
$$

where,

$\boldsymbol{E}_{r}^{0}$ : node voltage vector preceding the fault at fictitious bus $r . \quad \boldsymbol{E}_{r}^{0}=\left[E_{r 1}^{0}, E_{r 2}^{0}, E_{r 3}^{0}\right]^{T}$, with $\mathrm{T}$ denotes vector transpose;

$\boldsymbol{E}_{p 0}$ : node voltage vector preceding the fault at bus $p$. $\boldsymbol{E}_{p}^{0}=\left[E_{p 1}^{0}, E_{p 2}^{0}, E_{p 3}^{0}\right]^{T}$,

$E_{q 0}$ : node voltage vector preceding the fault at bus $q$. $\boldsymbol{E}_{q}=\left[E_{q 1}^{0}, E_{q 2}^{0}, E_{q 3}^{0}\right]^{T}$.

\subsubsection{Derivation of the driving point impedance at fault nodes and transfer impedance between fault nodes}

Remove all the sources in the network shown in Fig. 1. Then inject 1 Ampere current into node $\gamma_{i}$, where $i=1$, 2, 3 for a single circuit line, and $i=1,2, \ldots, 6$ for a double-circuit line. Applying KCL at bus $r$ yields

$$
\frac{\left(\boldsymbol{y}_{p r}+\boldsymbol{y}_{q r}\right)}{2} \boldsymbol{E}_{r}+\boldsymbol{z}_{p r}^{-1}\left(\boldsymbol{E}_{r}-\boldsymbol{E}_{p}\right)+\boldsymbol{z}_{q r}^{-1}\left(\boldsymbol{E}_{r}-\boldsymbol{E}_{q}\right)=\boldsymbol{u}_{i}
$$

where $\boldsymbol{u}_{i}$ is the $i t h$ column of a three by three or six by six identity matrix. From (9), $\boldsymbol{E}_{r}$ can be written as

$$
\boldsymbol{E}_{r}=\left[\frac{\left(\boldsymbol{y}_{p r}+\boldsymbol{y}_{q r}\right)}{2}+\boldsymbol{z}_{p r}^{-1}+\boldsymbol{z}_{q r}^{-1}\right]^{-1}\left(\boldsymbol{z}_{p r}^{-1} \boldsymbol{E}_{p}+\boldsymbol{z}_{q r}^{-1} \boldsymbol{E}_{q}+\boldsymbol{u}_{i}\right)
$$

Based on the definition, the value of the transfer impedance between a fault node and other fault nodes is equal to the voltage at other fault nodes, and the value 
of the driving point impedance at a fault node is equal to the voltage at the fault node [31]. Therefore, the values of $\boldsymbol{E}_{r}, \boldsymbol{E}_{p}$, and $\boldsymbol{E}_{q}$ are equal to $\boldsymbol{Z}_{r r_{i}}, \boldsymbol{Z}_{p r_{i}}$, and $\boldsymbol{Z}_{q r_{i}}$, respectively. Hence, the transfer impedance and driving point impedance at fault nodes are:

$$
\boldsymbol{Z}_{r r_{i}}=\left[\frac{\left(\boldsymbol{y}_{p r}+\boldsymbol{y}_{q r}\right)}{2}+\boldsymbol{z}_{p r}^{-1}+\boldsymbol{z}_{q r}^{-1}\right]^{-1} \cdot\left(\boldsymbol{z}_{p r}^{-1} \boldsymbol{Z}_{p r_{i}}+\boldsymbol{z}_{q r}^{-1} \boldsymbol{Z}_{q r_{i}}+\boldsymbol{u}_{i}\right)
$$

where,

$Z_{r r_{i}}=\left[Z_{r_{1} r_{i}}, Z_{r_{2} r_{i}}, Z_{r_{3} r_{i}}\right]^{T}$, with $\mathrm{T}$ denotes vector transpose;

$$
\begin{aligned}
Z_{p r_{i}} & =\left[Z_{p_{1} r_{i}}, Z_{p_{2} r_{i}}, Z_{p_{3} r_{i}}\right]^{T} ; \\
Z_{q r_{i}} & =\left[Z_{q_{1} r_{i}}, Z_{q_{2} r_{i}}, Z_{q_{3} r_{i}}\right]^{T} .
\end{aligned}
$$

$Z_{r_{1} r_{i}}, Z_{r_{2} r_{i}}, Z_{r_{3} r_{i}}$ : the transfer impedance between fault nodes and driving point impedance at fault nodes;

$Z_{p_{1} r_{i}}, Z_{p_{2} r_{i}}, Z_{p_{3} r_{i}}$ : the transfer impedance between nodes of bus $p$ and fault nodes;

$Z_{q_{1} r_{i}}, Z_{q_{2} r_{i}}, Z_{q_{3} r_{i}}$ : the transfer impedance between nodes of bus $q$ and fault nodes;

Setting $i=1,2,3$ for single circuit lines and $i=1,2, \ldots$, 6 for double-circuit lines will give all relevant driving point and transfer impedances related to fault nodes.

Similarly, as discussed in Section 3.1.1, it is revealed that these driving point and transfer impedances are functions of fault locations.

\subsection{Fault location algorithm}

This subsection presents the proposed fault location algorithm to locate multiple simultaneous faults in transmission systems. Figure 2 illustrates a scenario involving two simultaneous faults. A phase A to ground fault occurs at point $F_{1}$ on line segment $P_{1} Q_{1}$ with fault location $m_{1}$, and a phase $C$ to ground fault occurs simultaneously at point $F_{2}$ on line segment $P_{2} Q_{2}$ with fault location $m_{2}$.

Voltage measurements from specified locations are utilized to locate the unknown fault location $m_{1}$ and $m_{2}$. The voltage at any bus $l$ during the fault can be expressed as
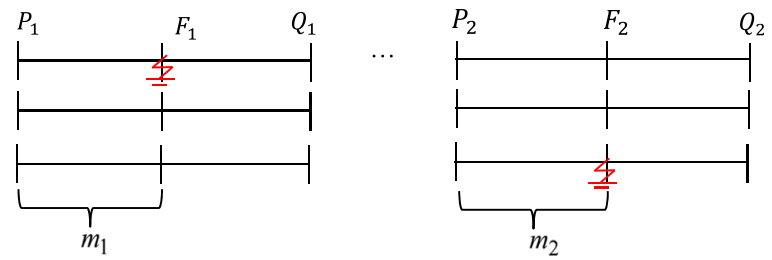

Fig. 2 Illustration of multiple simultaneous faults

$$
\boldsymbol{E}_{l}=\boldsymbol{E}_{l}^{0}-\left[\begin{array}{ll}
\boldsymbol{Z}_{l F_{1}} & \boldsymbol{Z}_{l F_{2}}
\end{array}\right]\left[\begin{array}{ll}
\boldsymbol{I}_{F_{1}} & \boldsymbol{I}_{F_{2}}
\end{array}\right]^{T}
$$

where,

$E_{l}$ : the voltage at bus $l$ during the fault;

$\boldsymbol{E}_{l}^{0}$ : the voltage at bus $l$ preceding the fault;

$Z_{l F_{1}}, Z_{l F_{2}}$ : the transfer impedance between bus $l$ and fault bus $F_{1}$, bus $l$ and fault bus $F_{2}$, respectively;

$\boldsymbol{I}_{F 1}, \boldsymbol{I}_{F 2}$ : the fault currents at the point $F_{1}$ and $F_{2}$.

Based on the measurements from two buses $L_{1}$ and $L_{2}$, The following two equations are obtained:

$$
\boldsymbol{E}_{L_{1}}=\boldsymbol{E}_{L_{1}}^{0}-\left[\begin{array}{ll}
\boldsymbol{Z}_{L_{1} F_{1}} & \boldsymbol{Z}_{L_{1} F_{2}}
\end{array}\right]\left[\begin{array}{ll}
\boldsymbol{I}_{F_{1}} & \boldsymbol{I}_{F_{2}}
\end{array}\right]^{T}
$$

$$
\boldsymbol{E}_{L_{2}}=\boldsymbol{E}_{L_{2}}^{0}-\left[\begin{array}{ll}
\boldsymbol{Z}_{L_{2} F_{1}} & \boldsymbol{Z}_{L_{2} F_{2}}
\end{array}\right]\left[\begin{array}{ll}
\boldsymbol{I}_{F_{1}} & \boldsymbol{I}_{F_{2}}
\end{array}\right]^{T}
$$

or in a compact format,

$$
\left[\begin{array}{c}
\boldsymbol{E}_{L_{1}} \\
\boldsymbol{E}_{L_{2}}
\end{array}\right]=\left[\begin{array}{c}
\boldsymbol{E}_{\mathrm{L}_{1}}^{0} \\
\boldsymbol{E}_{L_{2}}^{0}
\end{array}\right]-\left[\begin{array}{ll}
\boldsymbol{Z}_{L_{1} F_{1}} & \boldsymbol{Z}_{L_{1} F_{2}} \\
\boldsymbol{Z}_{L_{2} F_{1}} & \boldsymbol{Z}_{L_{2} F_{2}}
\end{array}\right]\left[\begin{array}{c}
\boldsymbol{I}_{F_{1}} \\
\boldsymbol{I}_{F_{2}}
\end{array}\right]
$$

where,

$\boldsymbol{E}_{L_{1}}, \boldsymbol{E}_{L_{2}}$ : the voltage during the fault at bus $L_{1}, L_{2}$, respectively;

$\boldsymbol{E}_{L_{1}}^{0}, \boldsymbol{E}_{L_{2}}^{0}$ : the voltage preceding the fault at bus $L_{1}, L_{2}$, respectively;

$Z_{L_{1} F_{1}}, Z_{L_{1} F_{2}}$ : transfer impedance matrix between bus $L_{1}$ and $F_{1}$, bus $L_{1}$ and $F_{2}$, respectively;

$Z_{L_{2} F_{1}}, Z_{L_{2} F_{2}}$ : transfer impedance matrix between bus $L_{2}$ and $F_{1}$, bus $L_{2}$ and $F_{2}$, respectively.

Equation (15) can be written in a more compact form as

$$
\boldsymbol{E}_{L_{1} L_{2}}=\boldsymbol{E}_{L_{1} L_{2}}^{0}-\boldsymbol{Z}_{L_{1} L_{2} F_{1} F_{2}} \boldsymbol{I}_{F_{1} F_{2}}
$$

The superimposed quantity, or the voltage change due to a fault, is

$$
\Delta \boldsymbol{E}_{L_{1} L_{2}}=-\boldsymbol{Z}_{L_{1} L_{2} F_{1} F_{2}} \boldsymbol{I}_{F_{1} F_{2}}
$$

From (17), the fault current vector is obtained as

$$
\boldsymbol{I}_{F_{1} F_{2}}=-\left(\boldsymbol{Z}_{L_{1} L_{2} F_{1} F_{2}}^{T} \boldsymbol{Z}_{L_{1} L_{2} F_{1} F_{2}}\right)^{-\mathbf{1}}\left(\boldsymbol{Z}_{L_{1} L_{2} F_{1} F_{2}}^{T} \Delta \boldsymbol{E}_{L_{1} L_{2}}\right)
$$

Furthermore, the voltage during the fault at fault buses are given by 


$$
\left[\begin{array}{c}
\boldsymbol{E}_{F_{1}} \\
\boldsymbol{E}_{F_{2}}
\end{array}\right]=\left[\begin{array}{l}
\boldsymbol{E}_{F_{1}}^{0} \\
\boldsymbol{E}_{F_{2}}^{0}
\end{array}\right]-\left[\begin{array}{ll}
\boldsymbol{Z}_{F_{1} F_{1}} & \boldsymbol{Z}_{F_{1} F_{2}} \\
\boldsymbol{Z}_{F_{2} F_{1}} & \boldsymbol{Z}_{F_{2} F_{2}}
\end{array}\right]\left[\begin{array}{c}
\boldsymbol{I}_{F_{1}} \\
\boldsymbol{I}_{F_{2}}
\end{array}\right]
$$

where,

$\boldsymbol{E}_{F_{1}}, \boldsymbol{E}_{F_{2}}$ : the voltage during the fault at fault bus $F_{1}$, $F_{2}$, respectively;

$\boldsymbol{E}_{F_{1}}^{0}, \boldsymbol{E}_{F_{2}}^{0}$ : the voltage preceding the fault at fault bus $F_{1}, F_{2}$, respectively;

$Z_{F_{1} F_{1}}, Z_{F_{2} F_{2}}$ : driving point impedance matrix at $F_{1}, F_{2}$, respectively;

$Z_{F_{1} F_{2}}, Z_{F_{2} F_{1}}$ : the transfer impedance matrix between $F_{1}$ and $F_{2}$.

Equation (19) can be written in a more compact form as

$$
\boldsymbol{E}_{F_{1} F_{2}}=\boldsymbol{E}_{F_{1} F_{2}}^{0}-\boldsymbol{Z}_{F_{1} F_{2} F_{1} F_{2}} \boldsymbol{I}_{F_{1} F_{2}}
$$

Based on (8), prefault voltages at the fault bus $F_{1}$ and $F_{2}$ can be expressed in terms of fault locations and the prefault voltages at the bus $P_{1}$ and $Q_{1}$, bus $P_{2}$ and $Q_{2}$. For example, $\boldsymbol{E}_{F_{1}}^{0}$ is derived as follows:

$\boldsymbol{E}_{F_{1}}^{0}=\left[\frac{\left(\boldsymbol{y}_{P_{1} F_{1}}+\boldsymbol{y}_{Q_{1} F_{1}}\right)}{2}+\boldsymbol{z}_{P_{1} F_{1}}^{-1}+\boldsymbol{z}_{Q_{1} F_{1}}^{-1}\right]^{-1} \cdot\left(\boldsymbol{z}_{P_{1} F_{1}}^{-1} \boldsymbol{E}_{P_{1}}^{0}+\boldsymbol{z}_{Q_{1} F_{1}}^{-1} \boldsymbol{E}_{Q_{1}}^{0}\right)$

where,

$\boldsymbol{z}_{P_{1} F_{1}}, \boldsymbol{z}_{Q_{1} F_{1}}$ : equivalent series impedance matrix of segment $P_{1} F_{1}$ and $Q_{1} F_{1}$;

$\boldsymbol{y}_{P_{1} F_{1}}, \boldsymbol{y}_{Q_{1} F_{1}}$ : equivalent shunt admittance matrix of segment $P_{1} F_{1}$ and $Q_{1} F_{1}$;

$\boldsymbol{E}_{P_{1}}^{0}, \boldsymbol{E}_{Q_{1}}^{0}$ : prefault voltages at $P_{1}$ and $Q_{1}$, which can be obtained by WAMS.

Since the fault resistances are purely resistive, the reactive power consumed by fault resistances at the two fault locations is zero, i.e.,

$$
\operatorname{Imag}\left(\left[\begin{array}{l}
\boldsymbol{E}_{F_{1}}^{T} \boldsymbol{I}_{F_{1}}^{*} \\
\boldsymbol{E}_{F_{2}}^{T} \boldsymbol{I}_{F_{2}}^{*}
\end{array}\right]\right)=0
$$

Solving (22) will yield the two unknown variables $m_{1}$ and $m_{2}$.

An alternative approach is described as follows when more measurements are available. Assuming that measurements from another two buses $L_{3}$ and $L_{4}$ are known. The following equation is obtained in a similar way to (18),

$$
\boldsymbol{I}_{F_{1} F_{2}}=-\left(\boldsymbol{Z}_{L_{3} L_{4} F_{1} F_{2}}^{T} \boldsymbol{Z}_{L_{3} L_{4} \mathrm{~F}_{1} F_{2}}\right)^{-\mathbf{1}}\left(\boldsymbol{Z}_{L_{3} L_{4} F_{1} F_{2}}^{T} \Delta \boldsymbol{E}_{L_{3} L_{4}}\right)
$$

Equating (18) and (23), it is obtained that

$$
\begin{aligned}
& \left(\boldsymbol{Z}_{L_{1} L_{2} F_{1} F_{2}}^{T} \boldsymbol{Z}_{L_{1} L_{2} F_{1} F_{2}}\right)^{-\mathbf{1}}\left(\boldsymbol{Z}_{L_{1} L_{2} F_{1} F_{2}}^{T} \Delta \boldsymbol{E}_{L_{1} L_{2}}\right) \\
= & \left(\boldsymbol{Z}_{L_{3} L_{4} F_{1} F_{2}}^{T} \boldsymbol{Z}_{L_{3} L_{4} F_{1} F_{2}}\right)^{-\mathbf{1}}\left(\boldsymbol{Z}_{L_{3} L_{4} F_{1} F_{2}}^{T} \Delta \boldsymbol{E}_{L_{3} L_{4}}\right)
\end{aligned}
$$

Equation (24) contains two unknown variables $m_{1}$ and $m_{2}$. Separating the equation into two real equations, from which the fault location can be obtained using Newton-Raphson method.

It is noted that the derivation does not assume the values of fault resistances, and thus the method is essentially immune to fault resistances.

\section{Case studies}

This section presents the evaluation results based on simulation studies. The developed fault zone identification method described in Section 2 and fault location algorithm proposed in Section 3 are applied to a 27-bus transmission system shown in Fig. 3.

In the figure, the lengths of transmission lines are shown in parentheses. In particular, the line between bus 9 and bus 10 is a long double-circuit transmission line.

The transmission system is modeled in Electromagnetic Transients Program (EMTP) [30] to obtain the measurements at each bus for fault scenarios with different fault locations, types, and resistances. The line is modelled based on distributed parameter line model and the system voltage and load level are considered to be typical transmission operating conditions.

The measurements obtained then are utilized to test the developed method and algorithm that are implemented in Matlab [32].

Section 4.1 presents the results for fault zone identification method, and Section 4.2 presents the results for fault location algorithm.

\subsection{Case studies for fault zone identification}

This subsection presents the results for fault zone identification method described in Section 2. The studied transmission system is partitioned into five protection zones as shown in Fig. 4. Due to the space limitation, the bus numbers, line lengths and the direction of currents are not labeled. To clarify the current directions, the rules used to select the direction of currents are explained as follows:

(1) It is natural to select the direction that current flows from a generator into a protection zone.

(2) For line currents that flow from bus to bus, the current direction is selected as from a smaller bus number to a larger bus number.

In this case study, the above rules are obeyed except that the current direction between bus 17 and bus 19 . 


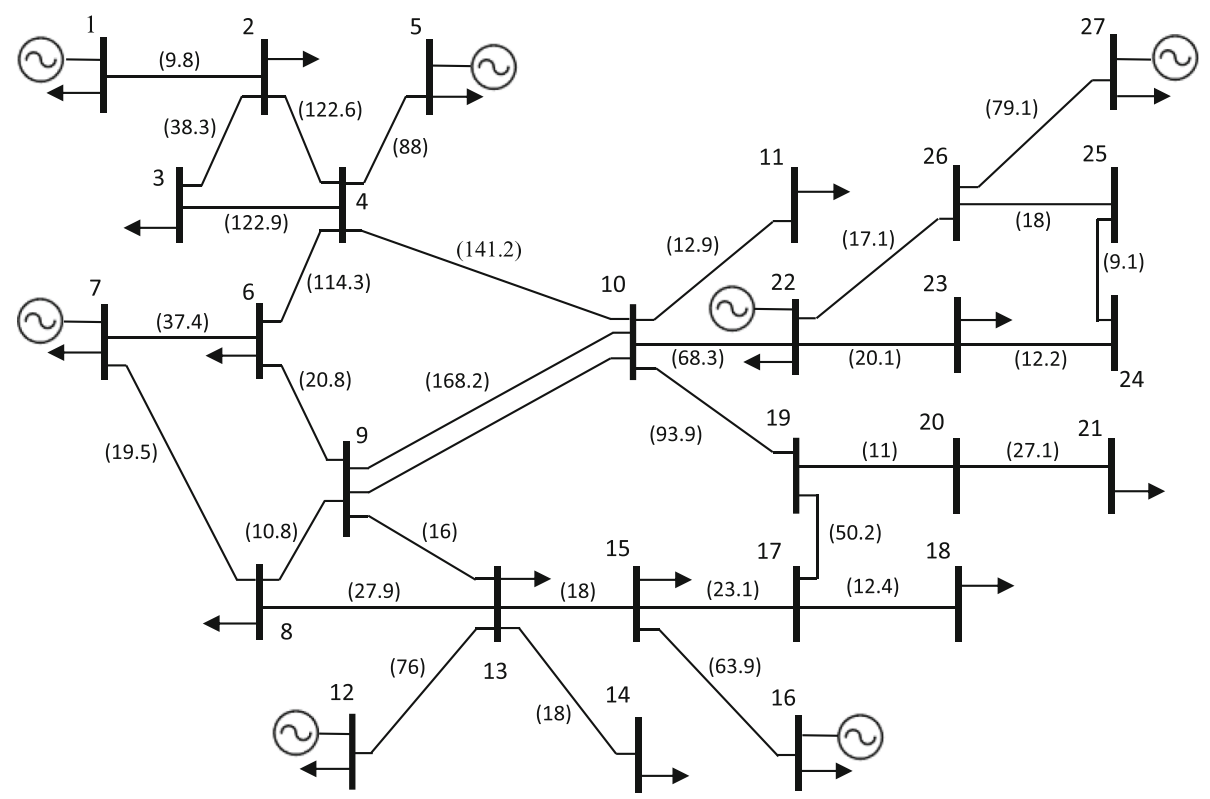

Fig. 3 The 27-bus transmission system used for analysis

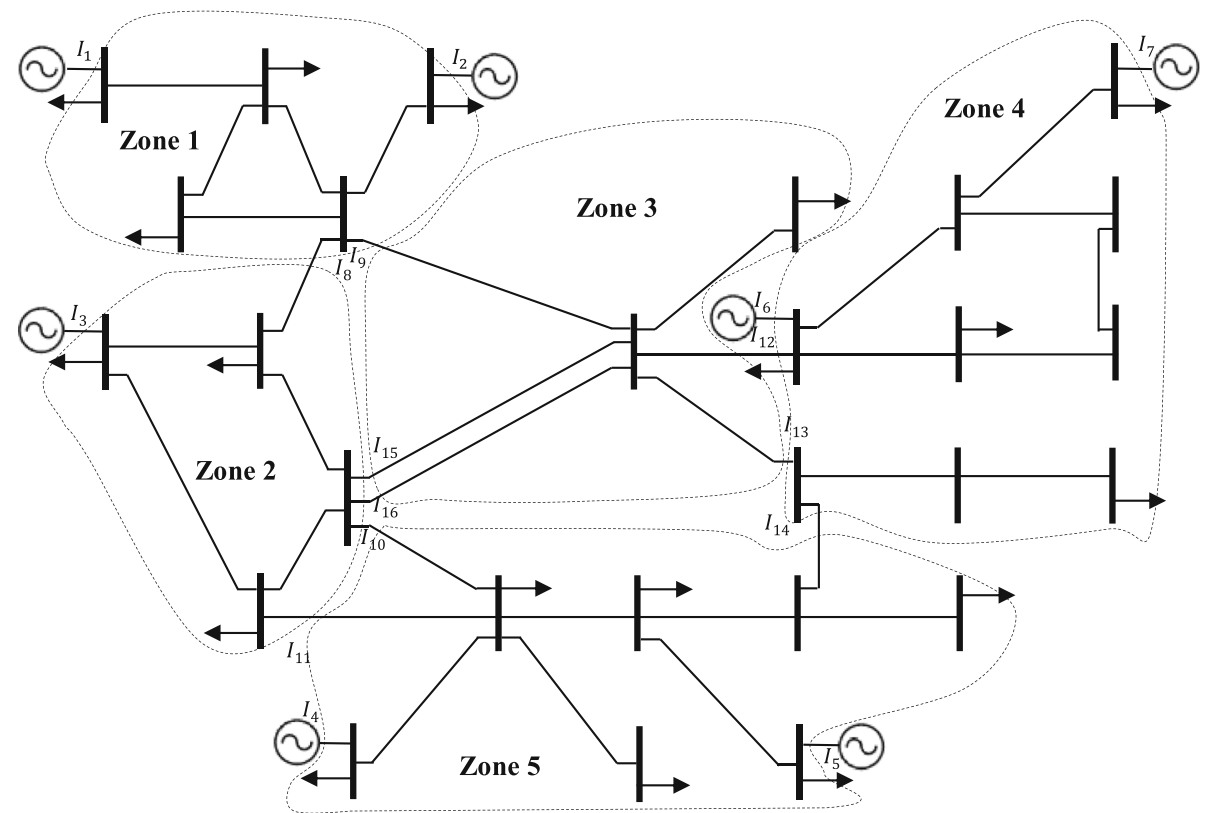

Fig. 4 Illustrating zone partition for fault zone identification 
Table 1 FZIV Preceding the fault

\begin{tabular}{llll}
\hline Zone number & $\boldsymbol{F Z I V}_{a}$ & $\boldsymbol{F Z I V}_{\boldsymbol{b}}$ & $\boldsymbol{F Z I V}_{\boldsymbol{c}}$ \\
\hline 1 & 3.606 & 3.606 & 3.606 \\
2 & 2.496 & 2.496 & 2.496 \\
3 & 4.224 & 4.224 & 4.224 \\
4 & 4.027 & 4.027 & 4.027 \\
5 & 4.653 & 4.653 & 4.653 \\
\hline
\end{tabular}

The fault zone identification method will be demonstrated in Section 4.1.1-4.1.3 for prefault, single fault, and simultaneous faults, respectively.

\subsubsection{Fault zone identification vector preceding the fault}

Based on the procedures described in Section 2, the following results are obtained:

The connection matrix $N$ is:

$$
N=\left[\begin{array}{ccccc}
1 & 0 & 0 & 0 & 0 \\
1 & 0 & 0 & 0 & 0 \\
0 & 1 & 0 & 0 & 0 \\
0 & 0 & 0 & 0 & 1 \\
0 & 0 & 0 & 0 & 1 \\
0 & 0 & 0 & 1 & 0 \\
0 & 0 & 0 & 1 & 0 \\
-1 & 1 & 0 & 0 & 0 \\
-1 & 0 & 1 & 0 & 0 \\
0 & -1 & 0 & 0 & 1 \\
0 & -1 & 0 & 0 & 1 \\
0 & 0 & -1 & 1 & 0 \\
0 & 0 & -1 & 1 & 0 \\
0 & 0 & 0 & -1 & 1 \\
0 & -1 & 1 & 0 & 0 \\
0 & -1 & 1 & 0 & 0
\end{array}\right]^{T}
$$

The current vector $\boldsymbol{I}$ is given by

$$
I=\left[\begin{array}{lllll}
I_{1} & I_{2} & I_{3} & \cdots & I_{16}
\end{array}\right]^{T}
$$

The fault zone identification vector $F Z I V$ is then obtained by substituting (25) and (26) into (1). The results of FZIV preceding the fault are summarized in Table 1. The first column of Table 1 lists the protection zone

Table 2 FZIV for a BG fault occurring in protection zone 2

\begin{tabular}{llll}
\hline Zone number & $\boldsymbol{F Z I V}_{a}$ & $\boldsymbol{F Z I V}_{\boldsymbol{b}}$ & $\boldsymbol{F Z I V}_{\boldsymbol{c}}$ \\
\hline 1 & 3.584 & 3.563 & 3.558 \\
2 & 3.257 & $\mathbf{1 2 . 9 1 9}$ & 3.433 \\
3 & 3.676 & 3.197 & 3.479 \\
4 & 4.006 & 3.983 & 3.949 \\
5 & 4.650 & 4.528 & 4.488 \\
\hline
\end{tabular}

The value corresponding to the faulted section and phase is written in bold font
Table 3 Difference between FZIV preceding the fault and during the $\mathrm{BG}$ fault occurring in protection zone 2

\begin{tabular}{llll}
\hline Zone number & $\left|\Delta \boldsymbol{F Z \boldsymbol { I }} \boldsymbol{V}_{a}\right|$ & $\left|\Delta \boldsymbol{F Z \boldsymbol { I }} \boldsymbol{V}_{b}\right|$ & $\left|\Delta \boldsymbol{F Z \boldsymbol { I }} \boldsymbol{V}_{c}\right|$ \\
\hline 1 & 0.022 & 0.043 & 0.048 \\
2 & 0.761 & $\mathbf{1 0 . 4 2 3}$ & 0.937 \\
3 & 0.548 & 1.027 & 0.745 \\
4 & 0.021 & 0.044 & 0.078 \\
5 & 0.003 & 0.125 & 0.165 \\
\hline
\end{tabular}

The value corresponding to the faulted section and phase is written in bold font

numbers. The rest columns of Table 1 list the values of FZIV for phase A, B, and C, respectively.

From Table 1, it is observed that the values of FZIV for each protection zone is quite small, indicating normal operation without any fault. Equal value for phases for each zone is expected because the transmission system is a balanced system preceding the fault.

Note that the connection matrix $N$ and current vector $I$ illustrated in this subsection are also applicable to latter subsections.

\subsubsection{Fault zone identification for a single fault}

Consider a Phase B to ground (BG) fault with fault resistance of $100 \Omega$ that occurs on the line between bus 6 and bus 9 . The measurements of bus 1 and bus 16 are utilized to calculate the FZIV. The results are summarized in Table 2. The first column of Table 2 lists the protection zone numbers. The rest columns of Table 2 list the values of FZIV for phase A, B, and C, respectively.

From Table 2, it is clear that the values of FZIV for phase $\mathrm{B}$ are significantly larger than other values in Table 2 . It is then determined that there is a fault involving phase $B$ occurring in protection zone 2 . Since the line between bus 6 and 9 is in protection zone 2, the result is expected.

Another approach to identifying the fault zone is to compare the values of FZIV preceding the fault and during the fault. For example, in Table 3, the last three columns list the absolute difference of FZIV for phase A, B, and $C$, respectively. It is evident that $\left|\Delta \boldsymbol{F} Z \boldsymbol{I} \boldsymbol{V}_{b}\right|$ for protection zone 2 is abnormally larger than other values in Table 3. Hence, a fault involving phase B in zone 2 can be determined to exist.

Table 4 FZIV for multiple simultaneous faults

\begin{tabular}{llll}
\hline Zone number & $\boldsymbol{F Z I V _ { a }}$ & $\boldsymbol{F Z I V _ { \boldsymbol { b } }}$ & $\boldsymbol{F Z I \boldsymbol { V } _ { \boldsymbol { c } }}$ \\
\hline 1 & 2.910 & 2.909 & 2.672 \\
2 & 7.612 & $\mathbf{2 5 . 5 0 5}$ & $\mathbf{2 7 . 8 4 8}$ \\
3 & $\mathbf{2 7 . 3 2 6}$ & $\mathbf{2 6 . 1 4 6}$ & $\mathbf{2 2 . 5 8 2}$ \\
4 & 2.407 & 2.439 & 2.230 \\
5 & 3.324 & 3.117 & 2.551 \\
\hline \multicolumn{7}{l}{ The value corresponding to the faulted section and phase is written in bold font }
\end{tabular}


Table 5 Difference between FZIV preceding the fault and during the multiple simultaneous faults

\begin{tabular}{llll}
\hline Zone number & $\left|\Delta F Z I V_{a}\right|$ & $\left|\Delta F Z I V_{b}\right|$ & $\left|\Delta F Z I V_{c}\right|$ \\
\hline 1 & 0.696 & 0.697 & 0.934 \\
2 & 5.116 & $\mathbf{2 3 . 0 0 9}$ & $\mathbf{2 5 . 3 5 2}$ \\
3 & $\mathbf{2 3 . 1 0 2}$ & $\mathbf{2 1 . 9 2 2}$ & $\mathbf{1 8 . 3 5 8}$ \\
4 & 1.620 & 1.588 & 1.797 \\
5 & 1.329 & 1.536 & 2.102 \\
\hline \multicolumn{7}{r}{ The value corresponding to the faulted section and phase is written in bold font }
\end{tabular}

This example demonstrates that the fault zone identification method is able to correctly identify the fault zone and faulted phase for a single fault.

\subsubsection{Fault zone identification for multiple simultaneous faults}

Impose a phase $B$ to phase $C(B C)$ fault with fault resistance of $15 \Omega$ on the line between bus 6 and bus 9 . In the meanwhile, impose an $\mathrm{ABC}$ fault with fault resistance of $1 \Omega$ on the double-circuit line between bus 9 and bus 10 . The measurements of bus 1,8 , and 16 are utilized to calculate the FZIV. The results are summarized in Table 4.

From Table 4, it is observed that $\boldsymbol{F Z I} \boldsymbol{V}_{\boldsymbol{b}}$ and $\boldsymbol{F Z I} \boldsymbol{V}_{\boldsymbol{c}}$ of protection zone 2, and $\boldsymbol{F Z I} \boldsymbol{V}_{\boldsymbol{a}}, \boldsymbol{F Z I} \boldsymbol{V}_{\boldsymbol{b}}$ and $\boldsymbol{F Z I} \boldsymbol{V}_{\boldsymbol{c}}$ of protection zone 3 are significantly larger than other values in Table 4. The observation indicates that there is a fault involving phase $B$ and $C$ falling in protection zone 2 and a second fault involving phase $\mathrm{A}, \mathrm{B}$, and $\mathrm{C}$ occurring in protection 3 .

The indication is accurate because the line between bus 6 and 9 is in protection zone 2, and the line between bus 9 and 10 is in protection zone 3 .

For the alternative approach, Table 5 presents the absolute difference of FZIV for phase A, B, and C, respectively. Table 5 shows that $\left|\Delta \boldsymbol{F Z I} \boldsymbol{V}_{b}\right|$ and $\left|\Delta \boldsymbol{F} \boldsymbol{Z} \boldsymbol{I} \boldsymbol{V}_{c}\right|$ for protection zone 2, and $\left|\Delta \boldsymbol{F} \boldsymbol{Z} \boldsymbol{I} \boldsymbol{V}_{a}\right|,\left|\Delta \boldsymbol{F} \boldsymbol{Z} \boldsymbol{I} \boldsymbol{V}_{b}\right|$ and $\left|\Delta \boldsymbol{F Z I} \boldsymbol{V}_{c}\right|$ for protection zone 3 are substantially larger than other values in the table. Thus, a simultaneous fault can be determined to be existing in the transmission system.

It is evidenced that the fault zone identification method not only identifies the fault zones, but also identifies faulted phases for multiple simultaneous faults.

In practical applications, $\Delta$ FZIV values are continuously calculated and compared with prefault FZIV values. For $|\triangle F Z I V|$, a threshold value of three times prefault FZIV value is chosen for fault and fault phase detection. Faults are determined to occur once the threshold value is exceeded. Then accurate location of faults is determined as follows.

\subsection{Case studies for fault location}

Once the fault zone is identified, the fault location will be pinpointed. In the study, the initial fault location is selected as 0.5 per unit for all cases. The fault location estimation accuracy is evaluated based on percentage error [18] as

$$
\% \text { Error }=\frac{\mid \text { Actual Location-Estimated Location } \mid}{\text { Total length of faulted line }} \cdot 100
$$

The fault location algorithm will be demonstrated in subsection 4.2.1 and 4.2.2 for single fault and simultaneous faults on transposed lines, respectively. Subsection 4.2.3 and 4.2.4 demonstrate the fault location results for single fault and simultaneous faults on untransposed lines, respectively. Various cases with different fault locations, types and fault resistances are simulated. Cases are typically found to reach convergence within three to eight iterations. Representative results are shown.

\subsubsection{Fault location for a single fault on transposed line}

Table 6 presents the fault location results. The first four columns of Table 6 list the faulted line segments, fault types, actual fault locations in per unit, and fault

Table 6 Fault location results for single faults on transposed lines

\begin{tabular}{llllll}
\hline $\begin{array}{l}\text { Faulted } \\
\text { lines }\end{array}$ & $\begin{array}{l}\text { Fault } \\
\text { types }\end{array}$ & $\begin{array}{l}\text { Actual FL } \\
\text { (p.u.) }\end{array}$ & $\begin{array}{l}\text { Fault Res. } \\
(\Omega)\end{array}$ & \multicolumn{2}{c}{\begin{tabular}{l} 
FL Est. error (\%) using data from selected buses \\
\cline { 5 - 6 }
\end{tabular}} \\
\hline $4-10$ & AG & 0.8 & 1 & 0.01 & 0.01 \\
$4-10$ & AG & 0.8 & 15 & 0.01 & 0.01 \\
$6-9$ & BC & 0.3 & 1 & 0.43 & 0.05 \\
$4-10$ & BC & 0.5 & 1 & 0.00 & 0.01 \\
$4-10$ & ABC & 0.2 & 1 & 0.00 & 0.00 \\
$4-10$ & BCG & 0.3 & 20 & 0.00 & 0.01 \\
$9-10$ & BG & 0.9 & 15 & 0.01 & 0.36 \\
$9-10$ & BC & 0.2 & 1 & 0.35 & 0.00 \\
$9-10$ & ABC & 0.7 & 1 & 0.01 & 0.12 \\
\hline
\end{tabular}


Table 7 Fault location results for multiple simultaneous faults on transposed lines

\begin{tabular}{|c|c|c|c|c|c|c|}
\hline \multirow[t]{2}{*}{ No. } & \multirow{2}{*}{$\begin{array}{l}\text { Fault } \\
\text { lines }\end{array}$} & \multirow{2}{*}{$\begin{array}{l}\text { Fault } \\
\text { types }\end{array}$} & \multirow{2}{*}{$\begin{array}{l}\text { Actual FL } \\
\text { (p.u.) }\end{array}$} & \multirow{2}{*}{$\begin{array}{l}\text { Fault Res. } \\
(\Omega)\end{array}$} & \multicolumn{2}{|c|}{ FL Est. error (\%) using data from selected buses } \\
\hline & & & & & $1 \& 8$ & $1,8 \& 16$ \\
\hline \multirow[t]{2}{*}{$1 s t$} & $4-10$ & $A G$ & 0.8 & 25 & 0.02 & 0.02 \\
\hline & $6-9$ & $B C$ & 0.3 & 10 & 0.49 & 0.40 \\
\hline \multirow[t]{2}{*}{ 2nd } & $4-10$ & AG & 0.8 & 50 & 0.01 & 0.00 \\
\hline & $9-10$ & BG & 0.9 & 25 & 0.02 & 0.01 \\
\hline \multirow[t]{2}{*}{$3 \mathrm{rd}$} & $6-9$ & $\mathrm{BC}$ & 0.3 & 1 & 0.05 & 0.07 \\
\hline & $9-10$ & BG & 0.9 & 15 & 0.01 & 0.01 \\
\hline
\end{tabular}

resistances in ohms, respectively. The last column of Table 6 lists the fault location estimation accuracy.

From Table 6, it is observed that quite accurate results are achieved under various fault conditions. Moreover, it should be noted that one bus measurement is enough to pinpoint the fault location for a single fault.

\subsubsection{Fault location for multiple simultaneous faults on transposed lines}

Table 7 presents the fault location results for simultaneous faults. All cases have a fault occurring on the double-circuit line between bus 9 and bus 10. Different fault types and fault resistances are considered. Voltage measurements from buses 1,8 , and 16 are utilized to estimate the fault locations.

The first column of Table 7 lists the case numbers. The second to fourth columns list the faulted lines, fault types, actual fault locations in per unit, and fault resistances in ohms, respectively. The last column lists the percentage error of estimated fault location.

The results demonstrate that quite accurate results are obtained by the proposed method, and the proposed method is able to handle simultaneous faults involving double-circuit line.

\subsubsection{Fault location for a single fault on untransposed line}

To emulate untransposed line, the line between bus 9 and 10 is made untransposed in this study. Table 8 tabulates fault location results for the untransposed double-circuit line between bus 9 and bus 10 under various fault conditions. Measurements at buses 1 and 16 are utilized to carry out the estimated fault locations.

From Table 8 , it is manifested that the estimation is quite accurate even for untransposed transmission lines. It is also noted that one measurement is enough to derive the fault location for single fault cases.

\subsubsection{Fault location for multiple simultaneous faults involving untransposed line}

In Table 9, fault location results for simultaneous faults that involve untransposed lines under diverse fault conditions are presented. Column 1 lists fault case number. Column 2 to 5 list actual fault conditions. The last two columns list the estimated fault location errors. It is important to note that the double circuit line between bus 9 and bus 10 is an untransposed line in this study.

Table 9 demonstrates that the proposed fault location algorithm yield accurate results and thus can deal with simultaneous faults involving untransposed lines.

\section{Conclusions}

Protection systems play a pivotal role in maintaining safety and reliability of electric power grids. This paper presents a system integrity protection scheme based on wide area monitoring system. The scheme is able to accurately identify fault zones and locate fault location

Table 8 Fault location results for single faults on untransposed line

\begin{tabular}{|c|c|c|c|c|c|}
\hline \multirow{2}{*}{$\begin{array}{l}\text { Faulted } \\
\text { lines }\end{array}$} & \multirow{2}{*}{$\begin{array}{l}\text { Fault } \\
\text { types }\end{array}$} & \multirow{2}{*}{$\begin{array}{l}\text { Actual FL } \\
\text { (p.u.) }\end{array}$} & \multirow{2}{*}{$\begin{array}{l}\text { Fault Res. } \\
(\Omega)\end{array}$} & \multicolumn{2}{|c|}{ FL Est. error (\%) using data from selected buses } \\
\hline & & & & 1 & $1 \& 16$ \\
\hline $9-10$ & $A G$ & 0.3 & 50 & 0.04 & 0.01 \\
\hline $9-10$ & AG & 0.8 & 1 & 0.01 & 0.25 \\
\hline $9-10$ & $\mathrm{BC}$ & 0.7 & 15 & 0.02 & 0.08 \\
\hline $9-10$ & $\mathrm{BC}$ & 0.2 & 1 & 0.34 & 0.01 \\
\hline $9-10$ & BCG & 0.4 & 20 & 0.04 & 0.02 \\
\hline $9-10$ & BCG & 0.6 & 1 & 0.02 & 0.01 \\
\hline $9-10$ & $A B C$ & 0.6 & 1 & 0.01 & 0.02 \\
\hline $9-10$ & $A B C$ & 0.7 & 20 & 0.01 & 0.12 \\
\hline
\end{tabular}


Table 9 Fault location results for multiple simultaneous faults involving untransposed line

\begin{tabular}{|c|c|c|c|c|c|c|}
\hline \multirow[t]{2}{*}{ No. } & \multirow{2}{*}{$\begin{array}{l}\text { Fault } \\
\text { lines }\end{array}$} & \multirow{2}{*}{$\begin{array}{l}\text { Fault } \\
\text { types }\end{array}$} & \multirow{2}{*}{$\begin{array}{l}\text { Actual FL } \\
\text { (p.u.) }\end{array}$} & \multirow{2}{*}{$\begin{array}{l}\text { Fault Res. } \\
(\Omega)\end{array}$} & \multicolumn{2}{|c|}{ FL Est. error (\%) using data from selected buses } \\
\hline & & & & & $1 \& 8$ & $1,8 \& 16$ \\
\hline \multirow[t]{2}{*}{$1 s t$} & $4-10$ & AG & 0.8 & 10 & 0.01 & 0.01 \\
\hline & $9-10$ & $\mathrm{BC}$ & 0.3 & 1 & 0.01 & 0.01 \\
\hline \multirow[t]{2}{*}{ 2nd } & $4-10$ & AG & 0.8 & 10 & 0.29 & 0.08 \\
\hline & $9-10$ & BG & 0.5 & 10 & 0.19 & 0.05 \\
\hline \multirow[t]{2}{*}{$3 \mathrm{rd}$} & $4-10$ & AG & 0.7 & 1 & 0.01 & 0.02 \\
\hline & $9-10$ & $B G$ & 0.4 & 20 & 0.03 & 0.04 \\
\hline
\end{tabular}

even in multiple, simultaneous fault scenarios. The algorithm is applicable to both transposed and untransposed transmission lines, which can be either single-circuit or double-circuit lines.

Case studies have shown that the fault zone identification method and fault location algorithm are accurate. The method and algorithm utilize wide area measurements, which are not necessarily at the terminals of faulted lines. Moreover, case studies show that no fault types are needed to identify the fault zones and pinpoint the fault locations.

\section{Acknowledgements}

None

\section{Funding}

None

\section{Availability of data and materials}

None

\section{Authors' contributions}

WF formulated the algorithm, performed the verification studies, and wrote the paper. YL provides guidance on algorithm development and writing. Both authors read and approved the final manuscript.

\section{Competing interests}

The authors declare that they have no competing interests.

Received: 16 November 2018 Accepted: 5 March 2019

Published online: 22 March 2019

\section{References}

1. Wang, S. Y., \& Dong, X. Z. (2011). A novel scheme for identification of lightning stroke and fault in EHV transmission lines. In International conference on advanced power system automation and protection (Vol. 3, pp. 2314-2319)

2. Wang, B., Dong, X., Bo, Z., \& Klimek, A. (2010). Negative-sequence pilot protection with applications in open-phase transmission lines. IEEE Transactions on Power Delivery, 25(3), 1306-1313.

3. Zhang, B. H., Hao, Z. G., \& Bo, Z. Q. (2016). New development in relay protection for smart grid. Protection and Control of Modern Power Systems. https://doi.org/10.1186/s41601-016-0025-x.

4. Reddy, M. J. B., \& Mohanta, D. K. (2017). Synchronized phasor measurements for smart grids. London: IET.

5. Begovic, M., Madani, V., \& Novosel, D. (2007). System integrity protection schemes (SIPS). In 2007 iREP Symposium on Bulk Power System Dynamics and Control - VII. Revitalizing Operational Reliability. Charleston: IEEE.

6. Hillberg, E., Trengereid, F., Breidablik, O., Uhlen, K., Kjolle, G., Lovlund, S., \& Gjerde, J. O. (2012). System integrity protection schemes - increasing operational security and system capacity. Paris: CIGRE.
7. McTaggart, C., Cardenas, J., Lopez, A., \& Bone, A. (2010). Improvements in power system integrity protection schemes. In 10th IET International Conference on Developments in Power System Protection. Manchester: IET.

8. Iliceto, F., Akansel, O., Akdeniz, M., Erikci, S., Korkmaz, Y., \& Ravikumar, K. (2018). System integrity protection schemes in the $400 \mathrm{kV}$ transmission network of Turkey. In 7th International Conference on Power System Protection and Automation. New Delhi: IEEE.

9. Pereira, C. E., \& Zanetta, L. C. (2004). Fault location in transmission lines using one-terminal postfault voltage data. IEEE Transaction on Power Delivery, 19(2), 570-575.

10. Apostolopoulos, C. A., \& Korres, G. N. (2011). A novel fault-location algorithm for double-circuit transmission lines without utilizing line parameters. IEEE Transaction on Power Delivery, 26(3), 1467-1478.

11. Roostaee, S., Thomas, M. S., \& Mehfuz, S. (2017). Experimental studies on impedance based fault location for long transmission lines. Protection and Control of Modern Power Systems. https://doi.org/10.1186/s41601-017-0048-y.

12. Lin, S., He, Z. Y., \& Li, X. P. (2012). Travelling wave time-frequency characteristic-based fault location method for transmission lines. IET Generation, Transmission \& Distribution, 6(8), 764-772.

13. Salehi, M., \& Namdari, F. (2018). Fault location on branched networks using mathematical morphology. IET Generation, Transmission \& Distribution, 12(1), 207-216.

14. Deng, F., Zeng, X. J., \& Pan, L. L. (2017). Research on multi-terminal traveling wave fault location method in complicated network based on cloud computing platform. Protection and Control of Modern Power Systems. https://doi.org/10.1186/s41601-017-0042-4.

15. Jamil, M., Sharma, S. K., \& Singh, R. (2015). Fault detection and classification in electrical power transmission system using artificial neural network. London: SpringerPlus. https://doi.org/10.1186/s40064-015-1080-x.

16. Koley, E., Verma, K., \& Ghosh, S. (2015). An improved fault detection classification and location scheme based on wavelet transform and artificial neural network for six phase transmission line using single end data only. SpringerPlus. https://doi.org/10.1186/s40064-015-1342-7.

17. Kawady, T., \& Stenzel, J. (2003). A practical fault location approach for double circuit transmission lines using single end data. IEEE Transactions on Power Delivery, 18(4), 1166-1173.

18. Kang, N., Chen, J., \& Liao, Y. (2015). A fault-location algorithm for seriescompensated double-circuit transmission lines using the distributed parameter line model. IEEE Transactions on Power Delivery, 30(1), 360-367.

19. Bo, Z. Q., Lin, X. N., Wang, Q. P., Yi, Y. H., \& Zhou, F. Q. (2016). Developments of power system protect and control. Protection and Control of Modern Power Systems. https://doi.org/10.1186/s41601-016-0012-2.

20. Rajaraman, P., Sundaravaradan, N. A., Mallikarjuna, B., Jaya, B. R. M., \& Mohanta, D. K. (2018). Robust fault analysis in transmission lines using synchrophasor measurements. Protection and Control of Modern Power Systems. https://doi.org/10.1186/s41601-018-0082-4.

21. Srivastava, A., Ravikumar, K., \& Zweigle, G. (2011). Wide-area monitoring and control using the real time digital simulator and a synchrophasor vector processor. International Transactions on Electrical Energy Systems, 21(4), 15211530.

22. Korkali, M., Lev-Ari, H., \& Abur, A. (2012). Traveling-wave-based fault-location technique for transmission grids via wide-area synchronized voltage measurements. IEEE Transaction on Power Systems, 27(2), 1003-1011.

23. Feng, G., \& Abur, A. (2016). Fault location using wide-area measurements and sparse estimation. IEEE Transaction on Power Systems, 31(4), 2938-2945. 
24. Hosseini, S. A., Sadeh, J., \& Mozafari, B. (2017). Wide-area fault location method considering gross measurement errors. IET Generation, Transmission \& Distribution, 11(18), 4670-4679.

25. Wang, Y., Li, W., \& Lu, J. (2010). Reliability analysis of wide-area measurement system. IEEE Transactions on Power Delivery, 25(3), 1483-1491.

26. Moller, J. G., Sorensen, M., Jóhansson, H., \& Ostergaard, J. (2017). Detecting topological errors with pre-estimation filtering of bad data in wide-area measurements. Manchester: IEEE Manchester PowerTech.

27. Dobakhshari, A. S., \& Ranjbar, A. M. (2015). A novel method for fault location of transmission lines by wide-area voltage measurements considering measurement errors. IEEE Transactions on Smart Grid, 6(2), 874-884.

28. Shahraeini, M., Ghazizadeh, M. S., \& Javidi, M. H. (2012). Co-optima placement of measurement devices and their related communication infrastructure in wide area measurement systems. IEEE Transactions on Smart Grid, 3(2), 684-691.

29. Grainger, J., \& Stevenson, W. (1994). Power system analysis (1st ed.p. 284). McGraw-Hill.

30. Leuven EMTP Centre. (1987). Alternative Transient Program. User Manual and Rule Book. Leuven: Leuven EMTP Centre

31. Liao, Y. (2013). Electric distribution system fault location considering shunt capacitances. Electric Power Components and Systems, 41(5), 519-536.

32. The MathWorks, Inc. (2016). Matlab help Manual (R2016b). Natick: The Mathworks, Inc.

\section{Submit your manuscript to a SpringerOpen ${ }^{\circ}$ journal and benefit from:}

- Convenient online submission

- Rigorous peer review

- Open access: articles freely available online

- High visibility within the field

- Retaining the copyright to your article

Submit your next manuscript at $\boldsymbol{\nabla}$ springeropen.com 